\title{
ON THE BERGMAN OPERATORS FOR LINEAR PARTIAL DIFFERENTIAL EQUATIONS
}

KAJ L. NIELSEN

1. Introduction. The frequent occurrence of the partial differential equation

$$
L(U)=U_{z z}+a U_{z}+b U_{z}+c U=0,
$$

where $a, b, c$ are functions of $z=x+i y$ and $\bar{z}=x-i y$ and $U_{z}=\partial U / \partial z$, $U_{\bar{z}}=\partial U / \partial \bar{z}, U_{z \bar{z}}=\partial^{2} U / \partial z \partial \bar{z}$, in the theory of dynamics has led to a considerable amount of investigation. This investigation has in part been directed along the lines of solving boundary value and characteristic value problems and of finding particular solutions. These problems are of course classical questions in the theory of partial differential equations and have been widely discussed in the literature. Among the recent developments on these problems is the work done by Bergman [1-5], ${ }^{1}$ who in his investigations has introduced operators

$$
P(f)=\int_{-1}^{+1} E(z, \bar{z}, t) f\left(z\left[1-t^{2}\right] / 2\right) d t /\left(1-t^{2}\right)^{1 / 2}
$$

where $f$ is an arbitrary analytic function of one complex variable. These operators shall be called the Bergman operators. They transform the class $A$ of analytic functions of one complex variable into a certain class of functions $\boldsymbol{C}(E)$, which has the following properties:

$\left(1^{\circ}\right)$ If $E$ is a solution of the equation

$$
\begin{aligned}
G(E)= & \left(1-t^{2}\right)\left(E_{z \bar{z}}+a E_{t}\right)-t^{-1}\left(E_{\bar{z}}+a E\right) \\
& +2 z t\left(E_{z \bar{z}}+a E_{z}+b E_{z}+c E\right)=0
\end{aligned}
$$

which satisfies certain conditions, then every function $U$ of the class $C(E)$ will be a particular solution of $L(U)=0$ and further there always exist solutions $E$ satisfying the mentioned conditions.

$\left(2^{\circ}\right)$ It is possible to determine two functions, say $E_{1}$ and $E_{2}$, so that $C\left(E_{1}\right)+C^{*}\left(E_{2}\right)$ represents the totality of solutions of $L(U)=0$ (see $[2,5]) . C^{*}$ is an analogous class of functions, the $f$ being an analytic function of $\bar{z}$.

The general problem of finding the Bergman operators for a given partial differential equation (1) has been thoroughly discussed by

Presented to the Society, November 26, 1943; received by the editors April 18, 1943.

1 The numbers in the brackets refer to the bibliography. 
Bergman $[1,2,3]$ and by the present author and Ramsay [7]. The singularities of the operators have been treated by Bergman [1] and the present author [6]. In [4] Bergman discussed a method of solution of the classical problems consisting of approximations by expressions $W_{n}=\sum_{\nu=1}^{n} \alpha_{\nu}^{(n)} \phi_{\nu}(x, y)$ where $\phi_{\nu}(x, y)$ are particular solutions of the considered differential equations. Here the $\alpha_{\nu}^{(n)}$ are constants which are to be determined by the requirements that the values of $W_{n}$ on the boundary approximate the given data. (This method is in a certain sense the reverse of the Rayleigh-Ritz method in which the approximating expressions satisfy the boundary conditions but do not satisfy the given equation.) The operators give a simple procedure for the construction of the particular solutions. To further expedite the numerical computation in practical problems it is desirable that the function $E(z, \bar{z}, t)$ have a simple form; for example,

$$
E(z, \bar{z}, t)=\exp \sum_{k=1}^{n} C_{k}(z, \bar{z}) t^{k} .
$$

The coefficients $a, b, c$, and $C_{k}(z, \bar{z})$ are connected by equation (3). This problem was considered in [7] and in the inyestigations which lead to that paper the converse problem suggested itself; namely, that of finding partial differential equations for which the Bergman operators have a particular form. In [7] it became apparent that a systematic approach to this converse problem would be to consider in turn expressions of the form exp $\left(N t^{n}\right)$, exp $\left(N t^{n}+M t^{m}\right)$, $\exp \left(N t^{n}+M t^{m}+R t^{r}\right)$, and so on, where $N, M$, and $R$ are functions of $z$ and $\bar{z}$. It is the purpose of this paper to give a general procedure for both problems and to complete the discussion for the case $E=\exp \left(N t^{n}+M t^{m}\right)$.

2. General procedure. The equation $L(U)=0$ can always be transformed into the equation

$$
L^{\prime}(V)=V_{z \bar{z}}+B V_{z}+C V=0
$$

(see $[2$, p. 1172]). Thus it suffices to consider this equation. The equation (3) reduces to

(6) $\quad G(E)=\left(1-t^{2}\right)\left(E_{t \bar{z}}\right)-E_{\bar{z}} / t+2 z t\left(E_{z \bar{z}}+B E_{\bar{z}}+C E\right)=0$.

A given form of $E$ will determine a particular solution

$$
V=P(f)=\int_{-1}^{+1} E(z, \bar{z}, t) f\left(z\left[1-t^{2}\right] / 2\right) d t /\left(1-t^{2}\right)^{1 / 2}
$$


of the partial differential equation $L^{\prime}(V)=0$ provided the coefficients $B$ and $C$ satisfy the equation (6) (see [2, p. 1171]). Thus for any given form of $E$ we determine the required derivatives

$$
E_{\bar{z}}, E_{z \bar{z}}, E_{t \bar{z}} \text {. }
$$

Substituting these values into (6) we obtain an equation which is zero for an arbitrary $t$; thus the coefficients of each power of $t$ must vanish. This yields a system of equations for $B, C, M$, and $N$. Upon solving this system we obtain the values for these unknowns and thus determine the partial differential equation (5) for which (7) with the given form of $E$ is a particular solution.

The converse problem then reduces to a discussion of this system. A systematic approach to such a discussion would be to consider in turn the cases in which $N$ is a function of $z$ only (that is, $N_{\bar{z}}=0$ ), $M$ is a function of $z$ only, and so on. If all functions $M, N$, and so on, are functions of $z$ only, then we have a trivial case. This is easily seen, for consider this case. We have

$$
E=\exp \sum_{\nu=1}^{n} C_{\nu}(z) t^{\nu}
$$

The required derivatives (8) for the equation (6) are then all zero as each has a derivative with respect to $\bar{z}$. If we further choose $C=0$, the equation (6) is satisfied. This would give the result that the function

$$
V=\int_{-1}^{+1}\left[\exp \sum_{\nu=1}^{n} C_{\nu}(z) t^{\nu}\right] f\left(z\left[1-t^{2}\right] / 2\right) d t /\left(1-t^{2}\right)^{1 / 2}
$$

is a particular solution of $L^{\prime}(V)=V_{z \bar{z}}+B(z, \bar{z}) V_{\bar{z}}=0$ for all values of $B(z, \bar{z}), C_{\nu}(z)$, and $\nu$. But this is evident, as any analytic function $f(z)$ is a solution of this partial differential equation. We shall therefore say that if $N_{\bar{z}}=0, M_{\bar{z}}=0$, and so on, simultaneously, we have a trivial case.

3. Case I. $E=\exp \left[N(z, \bar{z}) t^{n}\right]$. For simplicity of notation let $v=N(z$, $\bar{z}) t^{n}$. In general we shall let $v=\sum_{k=1}^{n} C_{k}(z, \bar{z}) t_{k}$ for each case; no ambiguity should arise in its meaning. The required derivatives (8) for the equation (6) are

$$
\begin{aligned}
E_{\bar{z}} & =e^{v}\left[N_{\bar{z}} t^{n}\right], \\
E_{t \bar{z}} & =e^{v}\left[n N N_{\bar{z}} t^{2 n-1}+n N_{\bar{z}} t^{n-1}\right], \\
E_{z \bar{z}} & =e^{v}\left[N_{z} N_{\bar{z}} t^{2 n}+N_{z \bar{z}} t^{n}\right] .
\end{aligned}
$$


The system of equations obtained by substituting (10) into (6) and setting the coefficients of the various powers of $t$ equal to zero is

$$
\begin{aligned}
t^{n-1}: & (n-1) N_{z}=0, \\
t^{2 n-1}: & n N N_{z}=0, \\
t^{n+1}: & (2 z B-n) N_{z}+2 z N_{z z}=0, \quad t: \quad 2 z C=0 .
\end{aligned}
$$

THEOREM 1. Exclusive of the trivial case, the only possible values of $n$ in Case I for which (7) is a particular solution of $L^{\prime}(V)=0$ are $n=0$, $n=1$, and $n=2$.

PRoof. To consider any case other than the trivial case $N$ must be a function of $\bar{z}$ as well as $z$; that is, $N_{z} \neq 0$. By (11) $N_{z} \neq 0$ only if $2 n-1=X$ where $X$ denotes any exponent of $t$ in (11). Thus

$$
\begin{array}{llll}
2 n-1=n-1 & \text { or } \quad n=0 ; & 2 n-1=2 n+1, \quad \text { impossible; } \\
2 n-1=n+1 & \text { or } \quad n=2 ; & 2 n-1=1 \text { or } n=1 . & \text { q.e.d. }
\end{array}
$$

Theorem 2. When $n=0$, Case I reduces to the trivial case.

PRoof. If $n=0$, the system (11) reduces to

$$
\begin{aligned}
t^{-1}: & -N_{z}=0, \\
t: & 2 z N_{z} N_{z}+2 z N_{z z}+2 B z N_{z}+2 z C=0 .
\end{aligned}
$$

From the first of these equations $N_{z}=0$ which is the trivial case.

The cases for $n=1$ and $n=2$ have been discussed in [7] and the solutions for $B, C$, and $N$ were obtained there.

4. Case II. $E=\exp \left[N t^{n}+M t^{m}\right]=e^{\natural}$. If $N=0$, or $M=0$, or $n=m$ this case reduces to Case I; thus we consider only $N \neq 0, M \neq 0$, $n \neq m$. In order to avoid further duplication we may, without any loss of generality, order $m$ and $n$ in the following manner

$$
n<m \text { if } n \text { and } m \text { have different signs; }
$$

$$
|n|>|m| \text { in all other cases. }
$$

The required derivatives $(8)$ are

$$
\begin{aligned}
E_{z}= & e^{v}\left[N_{z} t^{n}+M_{z} t^{m}\right], \\
E_{z} t= & e^{v}\left[m M_{z} t^{m-1}+m M_{z} t^{2 m-1}+n N_{z} t^{n-1}+n N N_{\bar{z}} t^{2 n-1}\right. \\
& \left.+\left(m M N_{z}+n N M_{z}\right) t^{n+m-1}\right], \\
E_{z z}= & e^{v}\left[M_{z z} t^{m}+M_{z} M_{z} t^{2 m}+N_{z z} t^{n}+N_{z} N_{z} t^{2 n}\right. \\
& \left.+\left(M_{z} N_{z}+M_{z} N_{z}\right) t^{n+m}\right] .
\end{aligned}
$$


The system of equations obtained by substituting (13) into (6) and setting the coefficients of the various powers of $t$ equal to zero is

$$
\begin{aligned}
t^{2 n-1}: & n N N_{z}=0, \\
t^{n-1}: & (n-1) N_{z}=0, \\
t^{2 n+1}: & \left(2 z N_{z}-n N\right) N_{z}=0, \\
t^{n+1}: & (2 z B-n) N_{z}+2 z N_{z z}=0, \\
t^{2 m-1}: & m M M_{z}=0, \\
t^{m-1}: & (m-1) M_{z}=0, \\
t^{2 m+1}: & \left(2 z M_{z}-m M\right) M_{z}=0, \\
t^{m+1}: & (2 z B-m) M_{z}+2 z M_{z \bar{z}}=0, \\
t^{n+m-1}: & m M N_{z}+n N M_{z}=0, \\
t^{n+m+1}: & \left(2 z M_{z}-m M\right) N_{z}+\left(2 z N_{z}-n N\right) M_{z}=0, \\
t: & 2 z C=0 .
\end{aligned}
$$

As was indicated in $\$ 2$ the procedure of analysis is to first consider $N_{z}=0, M_{z} \neq 0$; then $M_{z}=0, N_{z} \neq 0$; and finally neither equal to zero. If $N_{z}=0$ and $M_{z}=0$, we have of course the trivial case. From (14) it is clear that $N_{z}=0$ unless $2 n-1=X$ where $X$ denotes any exponent of $t$ in the system (14). Checking all these possibilities and using (12), we obtain the following property.

Property 1. $N_{z}=0$ for all values of $m$ and $n$ except $n=1$ and $n=2$.

If $N_{z}=0$, the system (14) reduces to

$$
\begin{aligned}
& t^{2 m-1}: \quad m M M_{z}=0 \text {, } \\
& t^{m-1}:(m-1) M_{z}=0, \quad t^{2 m+1}:\left(2 z M_{z}-m M\right) M_{z}=0 \text {, } \\
& \text { (15) } t^{m+1}:(2 z B-m) M_{z}+2 z M_{z i}=0, t^{n+m-1}: \quad n N M_{z}=0 \text {, } \\
& t^{n+m+1}:\left(2 z N_{z}-n\right) N M_{z}=0, \quad t: \quad 2 z C=0 .
\end{aligned}
$$

If $M_{3}=0$, the system (14) reduces to

$$
\begin{array}{rlrl}
t^{2 n-1}: & n N N_{z}=0, \\
t^{n-1}: & (n-1) N_{z}=0, & t^{2 n+1}: & \left(2 z N_{z}-n N\right) N_{z}=0, \\
t^{n+1}: & (2 z B-n) N_{z}=0, & t^{n+m-1}: & m M N_{z}=0, \\
t^{n+m+1}: & \left(2 z M_{z}-m M\right) N_{z}=0, \quad t: & 2 z C=0 .
\end{array}
$$

THEOREM 3. Case II reduces to the trivial case

(i) if $n<0$ and $m \geqq 0$; 
(ii) if $n<0$ and $m<0$;

(iii) if $m>2$;

(iv) if $n>2$ and $m=0$;

(v) if $n>3$ and $m=1$;

(vi) if $n>4$ and $m=2$;

(vii) if $n=3$ and $m=2$.

Proof. By definition of the trivial case it is necessary to show only that under these conditions $N_{z}=0$ and $M_{\bar{z}}=0$.

(i) and (ii). If $n<0, N_{\bar{z}}=0$ by Property 1 . The consideration then reduces to an analysis of the exponents of $t$ in (15). In (15) $M_{\bar{z}}=0$ unless $2 m-1=X$ and $n+m-1=X$ simultaneously, $X$ denoting any other exponent of $t$ in the system (15). If $n<0$ and $m \geqq 0,2 m-1=X$ only if $m=0, m=1$, or $m=2 ; n+m-1=X$ only if $m>2$. By a comparison it is easily seen that none of these conditions are satisfied simultaneously, therefore $M_{\bar{z}}$ is also zero. If $n<0$ and $m<0$, $n+m-1=X$ only if $n=m+2 ; 2 m-1=X$ only if $n=m-2$. These conditions can not be satisfied simultaneously, therefore $M_{\bar{z}}=0$.

(iii). In (14) $M_{\bar{z}}=0$ unless $m-1=X$; but by (i), (ii), and (12) this is impossible for $m>2$; thus $M_{\bar{z}}=0$. If $M_{\bar{z}}=0$, the system (14) reduces to (16) and $N_{\bar{z}}=0$ unless $2 n-1=X, n-1=X$, and $n+m-1=X$ simultaneously in (16). Checking these conditions one easily sees that this is impossible if $m>2$. Thus $N_{\bar{z}}$ is also equal to zero.

We now turn to (iv), (v), and (vi). If $n>2, N_{\bar{z}}=0$ by Property 1 . The system (14) then reduces to (15).

(iv). If $m=0$, the exponents of $t$ in (15) become

$$
-1,1, n-1 \text {, and } n+1 \text {. }
$$

Further $M_{\bar{z}}=0$ unless $-1=X$ and $n-1=X$ simultaneously. This is impossible if $n>2$. Therefore $M_{z}$ is also equal to zero.

(v). If $m=1$, the exponents of $t$ in (15) become

$$
1,3,2, n \text {, and } n+2 \text {. }
$$

Further $M_{\bar{z}}=0$ unless $n=X$ which is impossible if $n>3$. Therefore $M_{\bar{z}}$ is also zero.

(vi). If $m=2$, the exponents of $t$ in (15) become

$$
3,1,5, n+1, n+3 \text {. }
$$

Further $M_{\bar{z}}=0$ unless $n+1=X$ which is impossible if $n>4$. Therefore $M_{\bar{z}}$ is also zero.

(vii). If $n=3$ and $m=2$, it is easily seen that the system (14) yields $N_{z}=0$ and $M_{t}=0$. 
THEOREM 4. The only possible integral values for $m$ and $n$ which yield solutions different from those of the trivial case are

(i) $m=0$ and $n=1$ or $n=2$;

(ii) $m=1$ and $n=2$ or $n=3$;

(iii) $m=2$ and $n=4$.

Proof. This theorem follows directly from Theorem 3.

The cases of Theorem 5 have been discussed in [7] and solutions for $B, C, M$, and $N$ were obtained there.

\section{BIBLIOGRAPHY}

1. Stefan Bergman, Über Kurvenintegrale von Functionen zweier komplexen Veranderlichen, die die Differentialgleichung $\Delta V+V=0$ befriedigen, Math. Zeit. vol. 32 (1930) pp. 386-405.

2. Zur Theorie der Functionen, die eine lineare partielle Differentialgleichung befriedigen, Rec. Math. (Mat. Sbornik) N.S. vol. 2 (1937) pp. 1169-1197.

3. - Sur un lien entre la théorie des équations aux derivées partielles elliptiques et celle des fonctions d'une variable complexe, C. R. Acad. Sci. Paris vol. 205 (1937) pp. 1198-1200 and 1360-1362.

4. - The approximation of functions satisfying a linear partial differential equation, Duke Math. J. vol. 6 (1940) pp. 537-561.

5. Linear operators in the theory of partial differential equations, Trans. Amer. Math. Soc. vol. 53 (1943) pp. 130-155.

6. K. L. Nielsen, Some properties of functions satisfying partial differential equations of elliptic type, Duke Math. J. in the near future.

7. K. L. Nielsen and B. P. Ramsay, On particular solutions of linear partial differential equations, Bull. Amer. Math. Soc. vol. 49 (1943) pp. 156-162.

Louisiana State UnIversity 